\title{
Caracterización de algunas propiedades en tableros de corteza de Pinus pinaster Ait. y tableros de vermiculita expandida
}

\section{Characterization of some properties on boards produced with bark of "Pinus pinaster" Ait. and produced with expanded vermiculite}

O. González-Prieto $^{(*)}$, M. C. Touza ${ }^{(*)}$, G. Pereiro(**)

RESUMEN

La búsqueda de nuevas aplicaciones en el uso de los tableros requiere de la innovación en sus propiedades. Las mejoras en el aislamiento, en la regulación de las condiciones ambientales, en su resistencia al fuego y el incremento en sus propiedades físico-mecánicas, aumentan la diversificación de sus usos. El empleo de nuevas materias en los tableros y/o en los materiales compuestos, denominados composites (1), posibilita la mejora en estas propiedades. Esta publicación analiza diversas propiedades físicas y mecánicas de varios tipos de tableros monocapa, elaborados, por un lado, a base de partículas minerales de vermiculita expandida aglutinadas con silicatos de vidrios solubles (2), y por otro lado, a base de partículas lignocelulósicas de corteza de Pinus pinaster Ait., aglutinadas con cementos derivados del magnesio (3), incluidos ya estos últimos en la clasificación de la norma (4). Como material de referencia se emplean tableros comerciales de virutas de madera (5).

$850-14$

Palabras clave: tablero; corteza; vermiculita expandida; cemento de periclasa; vidrios solubles; propiedades físicas y mecánicas.

\section{SUMMARY}

The search for new applications to use boards requires innovation of their properties. The improvement of insulation properties, the possibility to regulate the environmental conditions, the improvement of fire resistance and the increase of physical and mechanical properties allow a more diverse use of boards. Using new components in the production of boards and/or component materials also called composites (1), enables the improvement of these properties. This publication analyses different physical and mechanical properties of various types of single-layer boards produced either based on expanded vermiculite particles bonded with soluble silicate glasses (2) or based on lignocelluloses particles bark of "Pinus pinaster" Ait., agglutinated with cement derivatives of magnesium (3), the latter included in the classification of the norm (4). Commercial wood wool boards (5) are used as reference material.
Keywords: board; bark; expanded vermiculite; periclasa cement; soluble glass; mechanical and physical properties. 
1. Morfología de los materiales de partida.

\section{INTRODUCCIÓN}

La demanda de soluciones más eficaces en el campo de la acústica arquitectónica y del aislamiento térmico está propiciando el empleo de materiales ligeros y de elevada permeabilidad. Al mismo tiempo, estos materiales deben optimizar la relación entre su densidad y su porosidad para poder potenciar las prestaciones térmicas y acústicas, sin penalizar en exceso su comportamiento mecánico.

Los materiales empleados en el presente estudio confieren a los tableros elaborados una elevada porosidad, lo que permite mejorar sus prestaciones finales de aislamiento térmico, absorción acústica y regulación de la humedad ambiental, dotándolos de propiedades interesantes para diversas aplicaciones (por ejemplo para el uso de placas de los falsos techos, como elementos de reIleno en las cámaras aislantes, etc.). Como referencia, paneles obtenidos a partir de materiales de biomasa aglomerados con magnesitas obtienen valores de conductividad térmica de 0,070 a 0,095 W/mK, mientras que paneles elaborados con vermiculita expandida alcanzan unos valores de conductividad térmica de $0,15 \mathrm{~W} / \mathrm{mK}(6$, 7 , 8). Por otro lado, las resinas tradicionalmente empleadas como aglomerantes en los tableros derivados de la madera, están siendo limitadas principalmente por las legislaciones en emisión de formaldehído (9) y tienden a ser sustituidas progresivamente por resinas naturales y/o compuestos inorgánicos como, por ejemplo, los cementos de magnesita y/o sus derivados (10).

En este contexto, el primer objetivo de este trabajo es analizar diversas propiedades físicas (densidad, humedad de equilibrio higroscópico e hinchazón en espesor tras 24 h de inmersión en agua) y mecánicas (flexión y resistencia a tracción perpendicular a las caras), en tableros elaborados con materiales porosos y con aglomerantes de compuestos inorgánicos, para comprobar si presentan un comportamiento adecuado en su empleo como materiales de construcción. Un segundo objetivo es analizar la influencia de la densidad en la variación de las propiedades estudiadas ya que este parámetro es uno de los principales factores que pueden ser regulados durante el proceso de fabricación.

Se comparan dos tipos de tableros elaborados según un proceso de prensado plano en caliente. Por un lado, tableros de corteza de pino marítimo (Pinus pinaster Ait.) elaborados con partículas de entre 2 y $8 \mathrm{~mm}$, y aglomeradas con cementos de magnesita. Por otro lado, dos tipos de tableros minerales elaborados con partículas de vermiculita expandida, aglomeradas con vidrios solubles. La vermiculita expandida se obtuvo mediante un tratamiento térmico que modifica la textura inicial de las partículas como consecuencia, principalmente, de la generación de vapor interlaminar, a través de su progresiva exfoliación entre planos internos (11 y 12). Las partículas adquieren de esta forma una geometría esencialmente laminar y un tamaño de entre 1 y 4 mm, en el caso de los tableros denominados "Vagalume", y una geometría de tipo granular con un tamaño de entre 0,2 y $1 \mathrm{~mm}$, en el caso de los tableros denominados "Corrubedo", estos últimos con un $10 \%$ más de aglomerante.

Como referencia se usaron tableros comerciales elaborados con virutas de madera de coníferas, de geometría esencialmente longitudinal, con anchos de entre 2 y $3 \mathrm{~mm}$ y largos superiores a $80 \mathrm{~mm}$, entrelazadas paralelamente a la superficie del tablero y recubiertas con un acabado superficial.

En la Figura 1 se muestran los materiales de partida que, una vez aglomerados, permiten obtener los tableros empleados en este trabajo. La búsqueda de la eficiencia energética en la edificación predispone el empleo de productos elaborados con materiales absorbentes porosos que incrementan sus propiedades aislantes (8) y permiten aportar soluciones importantes en el ámbito de la acústica arquitectónica (13). Una posible clasificación de los aislantes convencionales por su origen distinguiría, por un lado, los de naturaleza mineral -lanas de vidrio o de roca, vidrio celular, placas de yeso, perlita expandida, vermiculita, etc. - por otro lado, los de origen sintético-espumas de poliuretanos, poliestirenos expandidos o extruídos, etc. - y finalmente los de origen vegetal, básicamente corcho y fibras vegetales (14 y 15).

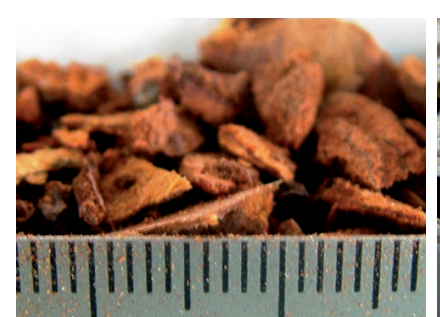

Partículas de corteza

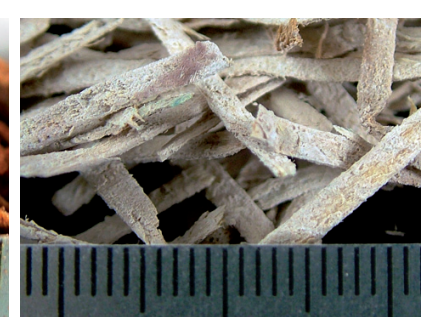

Virutas de madera

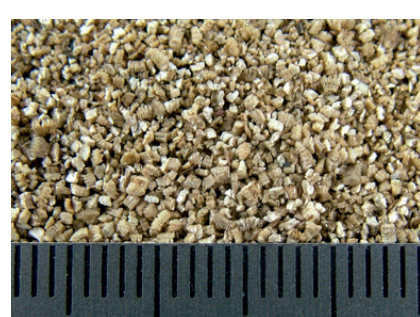

Vermiculita de "Corrubedo"

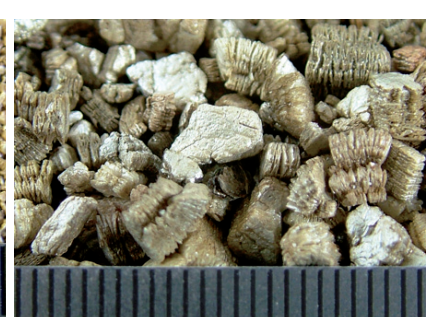

Vermiculita de "Vagalume" 
De esta forma, los tableros de vermiculita expandida caracterizados en el presente trabajo se posicionan como tableros aislantes e ignífugos con numerosas aplicaciones, entre las que cabe destacar soluciones técnicas de aislamiento térmico y acústico en materiales de construcción (8, 16 y 17$)$.

Con el fin de prever el comportamiento de los tableros en sus aplicaciones finales, donde cobran especial importancia aspectos como la regulación de la humedad relativa de los locales o la resistencia frente a los esfuerzos en su colocación y empleo (e peso propio del tablero, el peso de la luminarias o apliques, etc.) se han seleccionado como propiedades a ensayar la densidad, la hinchazón en espesor tras 24 horas de inmersión, la resistencia a flexión y la tracción perpendicular a la superficie de las caras.

La comparación de las diversas propiedades estudiadas entre ambos tipos de tableros minerales por un lado, y entre el tablero de corteza y el tablero de virutas de madera por otro lado, evidencian un incremento de las propiedades mecánicas al aumentar la densidad, además de mostrar cómo la geometría de las partículas de partida influye en las distintas propiedades estudiadas.

\section{PARTE EXPERIMENTAL}

Se han determinado cinco propiedades; densidad, humedad de equilibrio higroscópico (en condiciones de $20 \pm 2{ }^{\circ} \mathrm{C}$ y $65 \pm 5 \% \mathrm{Hr}$ ), hinchazón tras 24 horas de inmersión en agua, resistencia a la flexión y a la tracción perpendicular a las caras. Cada una de las propiedades se obtuvo ensayando 10 probetas, muestreadas de 4 tableros diferentes, obtenidos al azar de un lote de 500 tableros. Previamente a su ensayo, todas las probetas fueron estabilizadas en una cámara climática en a condiciones ambientales de $20 \pm$ $2^{\circ} \mathrm{C}$ de temperatura (T) y $65 \pm 5 \%$ de humedad relativa del aire $(\mathrm{Hr})$, de esta forma la condición higrotérmica de partida a efectos comparativos es la misma para todos los tableros.

La densidad $\rho$ (en $\left.\mathrm{Kg} / \mathrm{m}^{3}\right)$ se determinó conforme a la norma UNE EN 323:1994 (18), según la ecuación [1], donde $m$ es la masa de la probeta (en g), $b_{1}$ y $b_{2}$ son las medidas de sección y $t$ la medida de espesor (en m).

$$
\rho=\frac{m}{b_{1} \times b_{2} \times t} \times 10^{6}
$$

Para determinar la humedad de equilibrio higroscópico en las condiciones ambientales antes descritas, se emplearon probetas de dimensiones nominales $50 \times 50 \times 20 \mathrm{~mm}$ (excepto para los tableros de virutas de madera cuyo espesor nominal era de $15 \mathrm{~mm}$ ).
La humedad $H$, en \% sobre su peso anhidro, se determinó mediante la ecuación [2], donde $P_{h}$ es el peso de la probeta estabilizada en las condiciones antes descritas (en g) y $P_{0}$ es el peso (en g) tras permanecer en estufa a $103 \pm 2{ }^{\circ} \mathrm{C}$ hasta que dos pesadas consecutivas no difieran en más de un $0,1 \%$.

$$
\text { [2] } \quad H(\%)=\frac{P_{h}-P_{0}}{P_{0}} \times 100
$$

Para determinar la hinchazón tras 24 horas de inmersión en agua, se emplearon probetas con unas dimensiones nominales de $50 \times 50 \times 20 \mathrm{~mm}$ (excepto para los tableros de virutas con $15 \mathrm{~mm}$ de espesor) y se siguió el procedimiento descrito en la norma UNE EN 317:1994 (19). La hinchazón de cada probeta $G_{t}$, en \% sobre su espesor inicial, se determina según la ecuación [3], donde $t_{1}$ es el espesor antes de la inmersión (en $\mathrm{mm}$ ) y $t_{2}$ es el espesor después de inmersión (en mm).

$$
\text { [3] } \quad G_{t}=\frac{t_{2}-t_{1}}{t_{1}} \times 100
$$

La resistencia a flexión se determinó conforme a la norma UNE EN 310:1994 (20), mediante una máquina universal de ensayos y calculando la resistencia $\left(\mathrm{en} \mathrm{N} / \mathrm{mm}^{2}\right.$ ) según la ecuación [4], donde $F_{\text {máx }}$ es la carga máxima (en $N), L_{1}$ es la distancia entre apoyos (20 veces $t$, en $\mathrm{mm}$ ), $b$ es la anchura de la probeta $(50 \mathrm{~mm})$ y $t$ es el espesor de la probeta (en $\mathrm{mm}$ ).

$$
f_{m}=\frac{3 F_{\text {máx }} L_{1}}{2 b t^{2}}
$$

Para determinar la tracción perpendicular a las caras se emplearon probetas con unas dimensiones nominales de 50 x 50 x $20 \mathrm{~mm}$ (excepto para las tableros de virutas, con $15 \mathrm{~mm}$ de espesor) y se han seguido las indicaciones de la norma UNE EN 319:1994 (21). Las probetas se someten a un esfuerzo de tracción perpendicular a sus caras hasta la rotura. El valor de resistencia (en $\mathrm{N} / \mathrm{mm}^{2}$ ) se determina por la carga máxima aplicada en relación con la superficie de la probeta, según la ecuación [5] donde $F_{\text {máx }}$ es la carga de rotura (en N), $a$ y $b$ son las dimensiones de la sección de la probeta (en $\mathrm{mm})$.

$$
f_{t}=\frac{F_{\text {máx }}}{a \times b}
$$

Los perfiles de densidad bruta se obtuvieron mediante un perfilómetro de rayos $X$ (GRECOM Density Analyzer DA-X) sobre probetas de $50 \times 50 \mathrm{~mm}$. El perfil de densidad obtenido, que depende de la densidad del material inicial y del proceso de fabricación de los tableros, permite comprobar el grado de compactación interno de las partículas. 
2. Humedad de equilibrio higroscópico (\%). [Condiciones $20 \pm$ $\left.2^{\circ} \mathrm{C} / 65 \pm 5 \%\right]$.

3. Hinchazón en espesor (\%) 24h inmersión.

4. Resistencia a flexión $\left(\mathrm{N} / \mathrm{mm}^{2}\right)$.

5. Tracción perpendicular (N/ $\mathrm{mm}^{2}$ ).

\section{RESULTADOS Y DISCUSIÓN}

Se comparan, por un lado, las propiedades obtenidas al ensayar los tableros elaborados con partículas minerales de vermiculita expandida, y por otro lado, las obtenidas al ensayar los tableros elaborados con partículas de corteza y de virutas de madera. En esta comparativa, los valores medios obtenidos para la humedad de equilibrio higroscópico (Figura 2), en adelante $\mathrm{HEH}$, en unas condiciones ambientales de $20 \pm 2^{\circ} \mathrm{C}$ de temperatura y $65 \pm 5 \%$ de humedad relativa del aire, son de $18,74 \%(C V=0,04)$ para el tablero de corteza y de $12,05 \%(\mathrm{CV}=0,04)$ para el tablero de virutas de madera. En el caso de los tableros minerales, el valor medio de $\mathrm{HEH}$ ha sido de $8,66 \%(\mathrm{CV}=0,02)$ para el tablero Corrubedo y de $7,32 \%(C V=0,02)$ para el Vagalume. El valor de $\mathrm{HEH}$ al que se estabilizan los tableros de virutas coincide con los valores de referencia de otros tableros derivados de la madera como son los de virutas orientadas, conocidos por su denominación inglesa Oriented Strand Boards (OSB) (22). Por otro lado, las partículas de vermiculita han sufrido una expansión durante su proceso de fabricación, de unas 8 a 30 veces su tamaño original, teniendo mayor tamaño cuanto mayor ha sido su exfoliación. La mayor facilidad de captar moléculas de vapor de agua en sus partículas menos exfoliadas y de geometría más granular (23), justificaría los valores superiores en el tablero Corrubedo frente al Vagalume.
El valor medio de hinchazón de 3,35\% (CV $=0,39$ ) para el tablero de corteza, es inferior al del tablero de virutas, 4,96\% (CV = $0,11)$, siendo además su variabilidad mayor (Figura 3). Las propiedades hidrófobas de las partículas de corteza como consecuencia de su alto contenido en suberina y otros extractos (24) limitarían su afinidad frente al agua líquida (25), en contraposición a las fibras de madera. En el tablero mineral Corrubedo el valor medio de hinchazón es de $2,09 \%(C V=0,27)$, inferior al valor medio de los tableros Vagalume de 3,88\% (CV $=0,19$ ). En este resultado podría influir la mayor cantidad de aglomerante de vidrios solubles aplicada, un $10 \%$ en peso superior al aplicado en el tablero Vagalume. Los valores medios obtenidos muestran que los comportamientos frente al vapor de agua son contrapuestos a los obtenidos frente al agua líquida (Figura 2 y 3 ).

En el caso de la resistencia a flexión [Figura $4]$ el valor medio obtenido en los tableros de virutas, de $2,32 \mathrm{~N} / \mathrm{mm}^{2}(\mathrm{CV}=0,25)$, es más del triple del obtenido por los tableros de corteza, de $0,73 \mathrm{~N} / \mathrm{mm}^{2}(\mathrm{CV}=0,29)$. Sobre la base de estudios previos realizados en otros tipos de tableros (26 y 27), la geometría de las partículas granulares empleadas en el tablero de corteza, mucho menos esbeltas que las virutas de madera con geometría claramente longitudinal, justificaría este comportamiento. Asimismo, el recubrimiento superficial aplicado en el tablero
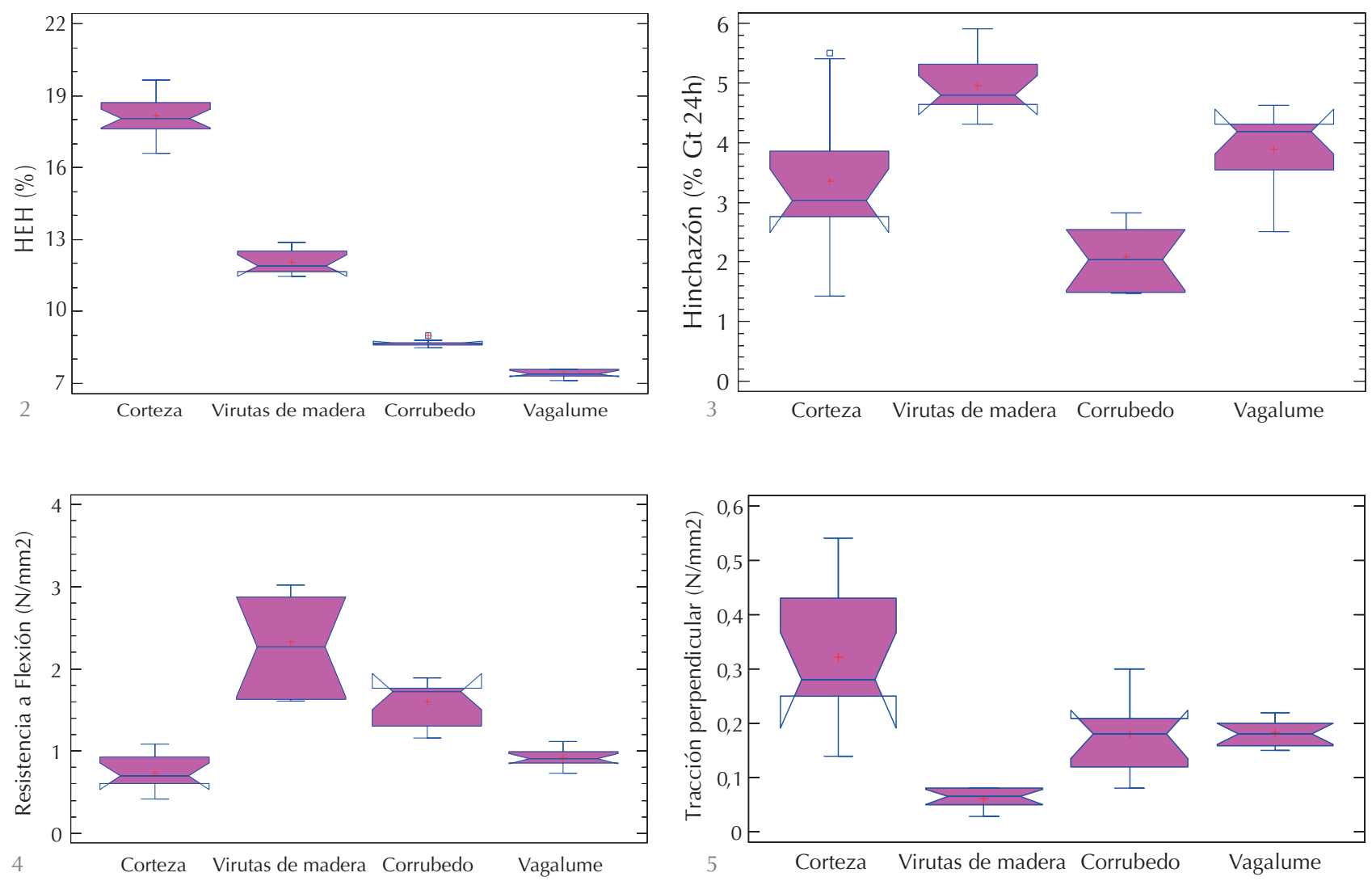
de virutas puede favorecer el incremento de esta propiedad. Un aumento en el aglutinante, favorecería el incremento de la resistencia a flexión (28). Esto podría justificar que los tableros minerales Corrubedo, con un $10 \%$ más de aglomerante y con una mayor densidad, tienen un valor de resistencia a flexión un $73 \%$ superior al tablero Vagalume, $1,59 \mathrm{~N} / \mathrm{mm}^{2}(\mathrm{CV}=0,16)$ frente a 0,92 $\mathrm{N} / \mathrm{mm}^{2}(\mathrm{CV}=0,13)$.

La resistencia a tracción perpendicular a las caras (Figura 5) es aproximadamente un $18 \%$ menor para el tablero de virutas que para el de corteza. Al igual que sucede con los tableros de partículas de madera (29), este resultado podría justificarse considerando que al reducir sustancialmente el grado de esbeltez de las partículas se mejora la cohesión en la parte central del tablero. Al mismo tiempo, a diferencia de los aglutinantes tradicionales a base de resinas derivadas del petróleo, los cementos no producen uniones químicas con las partículas lignocelulósicas (30), por lo que la matriz resistente del cemento tiende a ser más continua y resistente con partículas menos esbeltas. En los tableros minerales el valor medio de tracción prácticamente se equipara, con $0,18 \mathrm{~N} / \mathrm{mm}^{2}(\mathrm{CV}=0,12)$ para el tablero Vagalume y $0,17 \mathrm{~N} / \mathrm{mm}^{2}(\mathrm{CV}=$ $0,33)$ para el tablero Corrubedo.

\section{Comparativa entre tableros de corteza y de virutas}

La HEH media, en condiciones de $20 \pm 2{ }^{\circ} \mathrm{C}$ de temperatura y $65 \pm 5 \%$ de humedad relativa del aire, es superior en todos los valores obtenidos en el tablero de corteza frente al tablero de virutas (Figura 6 y Tabla 1). En relación al valor medio de hinchazón, en el tablero de virutas de madera se reduce al aumentar la densidad, mientras que los valores obtenidos para las probetas del tablero de corteza tienen una variabilidad mayor (Figura 7 y Tabla 2). La resistencia a flexión (Figura 8 y Tabla 3) y la resistencia a tracción (Figura 9 y Tabla 4) se incrementan al aumentar la densidad en ambos tipos de tableros.

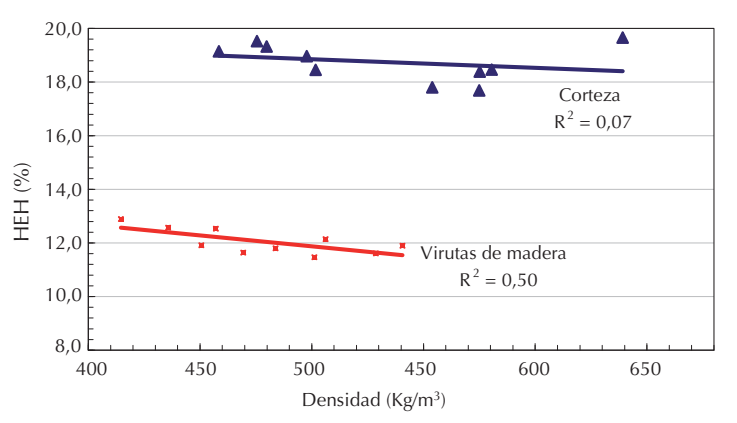

Tabla 1.

Valores HEH de los tableros de corteza y de virutas de madera.

\begin{tabular}{|l|r|r|r|r|}
\cline { 2 - 5 } \multicolumn{1}{c|}{} & \multicolumn{2}{c|}{ Corteza } & \multicolumn{2}{c|}{ Virutas de madera } \\
\cline { 2 - 5 } \multicolumn{1}{c|}{} & HEH (\%) & Densidad $\left(\mathrm{kg} / \mathrm{m}^{3}\right)$ & HEH $(\%)$ & Densidad $\left(\mathrm{kg} / \mathrm{m}^{3}\right)$ \\
\hline Media & 18,74 & 533,69 & 12,05 & 478,79 \\
\hline$\sigma$ & 0,69 & 59,06 & 0,47 & 40,68 \\
\hline CV & 0,04 & 0,11 & 0,04 & 0,08 \\
\hline
\end{tabular}

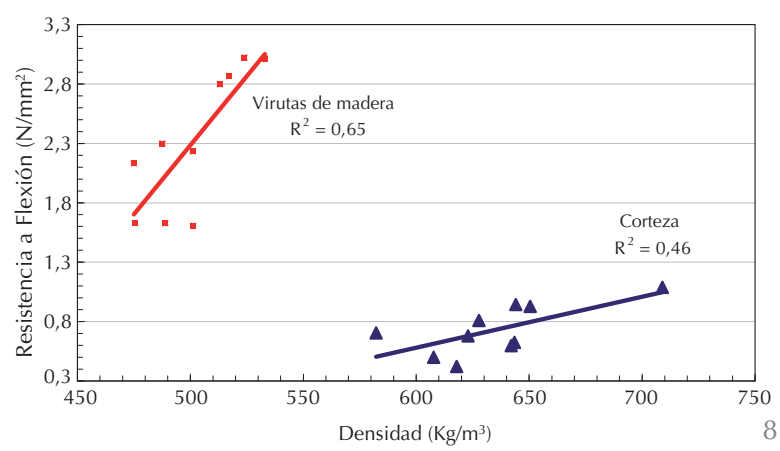

Tabla 3.

Valores de resistencia a flexión obtenidos para el tablero de corteza y el tablero de virutas de madera

\begin{tabular}{|l|r|r|r|r|}
\cline { 2 - 5 } \multicolumn{1}{c|}{} & \multicolumn{2}{c|}{ Corteza } & \multicolumn{2}{c|}{ Virutas de madera } \\
\cline { 2 - 5 } \multicolumn{1}{c|}{} & $\begin{array}{c}\text { R.flexión } \\
\left(\mathrm{N} / \mathrm{mm}^{2}\right)\end{array}$ & Densidad $\left(\mathrm{kg} / \mathrm{m}^{3}\right)$ & $\begin{array}{l}\text { R.flexión } \\
\left(\mathrm{N} / \mathrm{mm}^{2}\right)\end{array}$ & Densidad $\left(\mathrm{kg} / \mathrm{m}^{3}\right)$ \\
\hline$\sigma$ & 0,73 & 634,75 & 2,32 & 501,61 \\
\hline $\mathrm{CV}$ & 0,21 & 33,21 & 0,58 & 20,01 \\
\hline
\end{tabular}

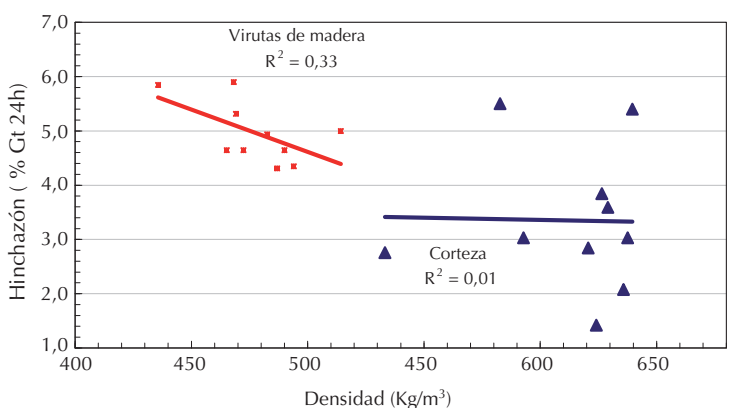

Tabla 2.

Valores de Hinchazón tras 24 horas de inmersión de los tableros de corteza y de virutas de madera

\begin{tabular}{|l|r|r|r|r|}
\cline { 2 - 5 } \multicolumn{1}{c|}{} & \multicolumn{2}{c|}{ Corteza } & \multicolumn{2}{c|}{ Virutas de madera } \\
\cline { 2 - 5 } \multicolumn{1}{c|}{} & Hinchazón (\%) & Densidad $\left(\mathrm{kg} / \mathrm{m}^{3}\right)$ & Hinchazón (\%) & Densidad $\left(\mathrm{kg} / \mathrm{m}^{3}\right)$ \\
\hline Media & 3,35 & 612,18 & 4,96 & 477,83 \\
\hline$\sigma$ & 1,30 & 33,55 & 0,57 & 21,01 \\
\hline $\mathrm{CV}$ & 0,39 & 0,05 & 0,11 & 0,04 \\
\hline
\end{tabular}

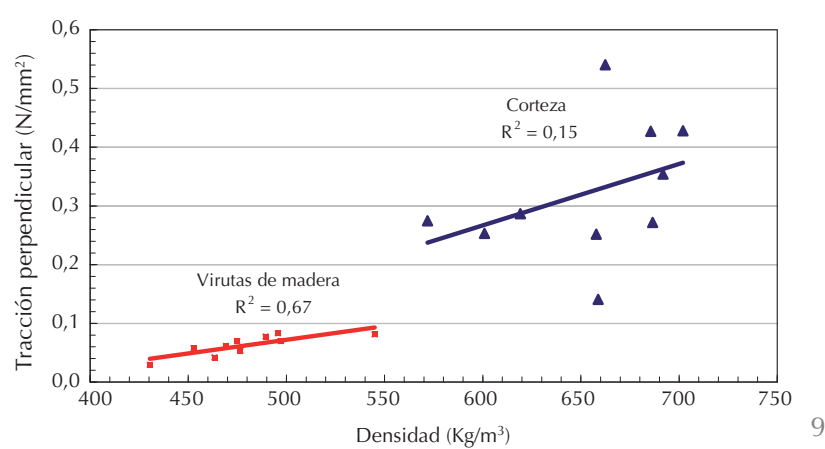

Tabla 4.

Valores de tracción perpendicular a las caras para los tableros de corteza y de virutas de madera

\begin{tabular}{|l|r|r|r|r|}
\cline { 2 - 5 } \multicolumn{1}{c|}{} & \multicolumn{3}{c|}{ Corteza } & \multicolumn{2}{c|}{ Virutas de madera } \\
\cline { 2 - 5 } \multicolumn{1}{c|}{} & $\begin{array}{c}\text { Tracción } \\
\left(\mathrm{N} / \mathrm{mm}^{2}\right)\end{array}$ & Densidad $\left(\mathrm{kg} / \mathrm{m}^{3}\right)$ & $\begin{array}{c}\text { Tracción } \\
\left(\mathrm{N} / \mathrm{mm}^{2}\right)\end{array}$ & Densidad $\left(\mathrm{kg} / \mathrm{m}^{3}\right)$ \\
\hline Media & 0,32 & 653,70 & 0,06 & 479,42 \\
\hline$\sigma$ & 0,12 & 43,05 & 0,02 & 30,68 \\
\hline $\mathrm{CV}$ & 0,36 & 0,07 & 0,28 & 0,06 \\
\hline
\end{tabular}


10. HEH (\%). [Condiciones $20 \pm$ $\left.2{ }^{\circ} \mathrm{C} / 65 \pm 5 \%\right]$.

11. Hinchazón en espesor (\%) 24 h inmersión.

12. Resistencia a flexión $\left(\mathrm{N} / \mathrm{mm}^{2}\right)$. 13. Tracción perpendicular $\left(\mathrm{N} / \mathrm{mm}^{2}\right)$.

\section{Comparativa entre tableros minerales}

La HEH media, en condiciones de $20 \pm$ $2{ }^{\circ} \mathrm{C}$ de temperatura y $65 \pm 5 \%$ de humedad relativa del aire, es un $38 \%$ superior en los tableros Corrubedo frente a los Vagalume (Figura 10 y Tabla 5) y en ambos no se observa una relación clara con el incremento de densidad. derando la premisa de a mayor exfoliación, mayor tamaño de partículas, los espacios porosos disponibles para las moléculas de agua son mayores en las partículas de los tableros Vagalume frente a los Corrubedo. Trabajos previos empleando selladores porosos han concluido que al reducir o limitar la porosidad disponible, la vermiculita reduce su capacidad de captación de agua líquida (10).
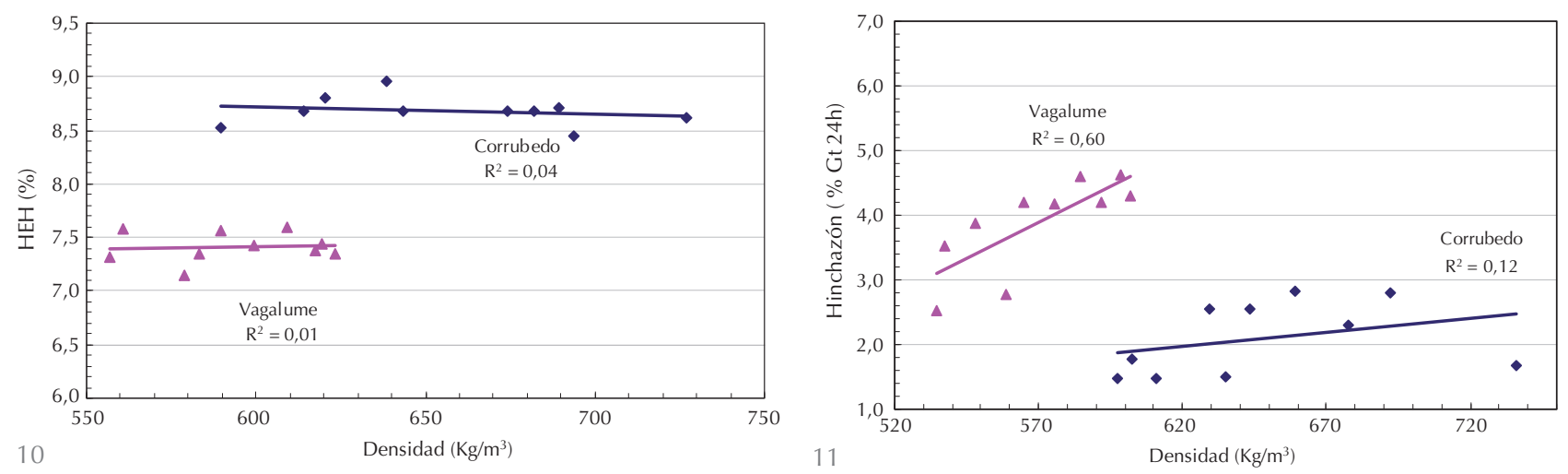

Tabla 6.

Tabla 5.

Valores HEH de los tableros de Vagalume y de Corrubedo

\begin{tabular}{|l|r|r|r|r|}
\cline { 2 - 5 } \multicolumn{1}{c|}{} & \multicolumn{2}{c|}{ Vagalume } & \multicolumn{2}{c|}{ Corrubedo } \\
\cline { 2 - 5 } \multicolumn{1}{c|}{} & HEH $(\%)$ & Densidad $\left(\mathrm{kg} / \mathrm{m}^{3}\right)$ & HEH $(\%)$ & Densidad $\left(\mathrm{kg} / \mathrm{m}^{3}\right)$ \\
\hline Media & 7,32 & 593,81 & 8,66 & 657,14 \\
\hline$\sigma$ & 0,14 & 23,87 & 0,14 & 42,79 \\
\hline CV & 0,02 & 0,04 & 0,02 & 0,07 \\
\hline
\end{tabular}

Valores de Hinchazón tras 24 horas de inmersión de los tableros de Vagalume y de Corrubedo

\begin{tabular}{|l|r|r|r|r|}
\cline { 2 - 5 } \multicolumn{1}{c|}{} & \multicolumn{2}{c|}{ Vagalume } & \multicolumn{2}{c|}{ Corrubedo } \\
\cline { 2 - 5 } \multicolumn{1}{c|}{} & Hinchazón (\%) & Densidad $\left(\mathrm{kg} / \mathrm{m}^{3}\right)$ & Hinchazón $(\%)$ & Densidad $\left(\mathrm{kg} / \mathrm{m}^{3}\right)$ \\
\hline Media & 3,88 & 569,59 & 2,09 & 638,54 \\
\hline$\sigma$ & 0,73 & 24,78 & 0,56 & 33,00 \\
\hline CV & 0,19 & 0,04 & 0,27 & 0,05 \\
\hline
\end{tabular}

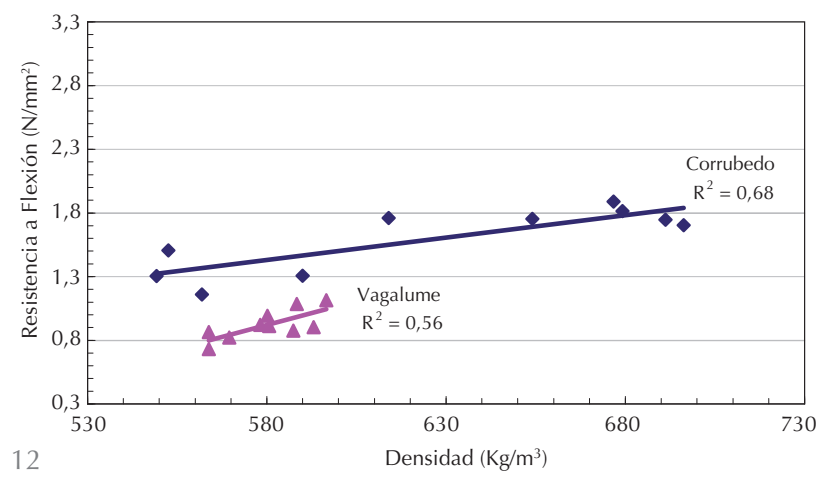

Tabla 7.

Valores de resistencia a flexión obtenidos para el tablero de Vagalume y el tablero de Corrubedo

\begin{tabular}{|l|r|r|r|r|}
\cline { 2 - 5 } \multicolumn{1}{c|}{} & \multicolumn{2}{c|}{ Vagalume } & \multicolumn{2}{c|}{ Corrubedo } \\
\cline { 2 - 5 } \multicolumn{1}{c|}{} & $\begin{array}{c}\text { R.flexión } \\
\left(\mathrm{N} / \mathrm{mm}^{2}\right)\end{array}$ & Densidad $\left(\mathrm{kg} / \mathrm{m}^{3}\right)$ & $\begin{array}{r}\text { R.flexión } \\
\left(\mathrm{N} / \mathrm{mm}^{2}\right)\end{array}$ & Densidad $\left(\mathrm{kg} / \mathrm{m}^{3}\right)$ \\
\hline Media & 0,92 & 580,15 & 1,59 & 626,51 \\
\hline$\sigma$ & 0,12 & 11,57 & 0,26 & 59,84 \\
\hline $\mathrm{CV}$ & 0,13 & 0,02 & 0,16 & 0,10 \\
\hline
\end{tabular}

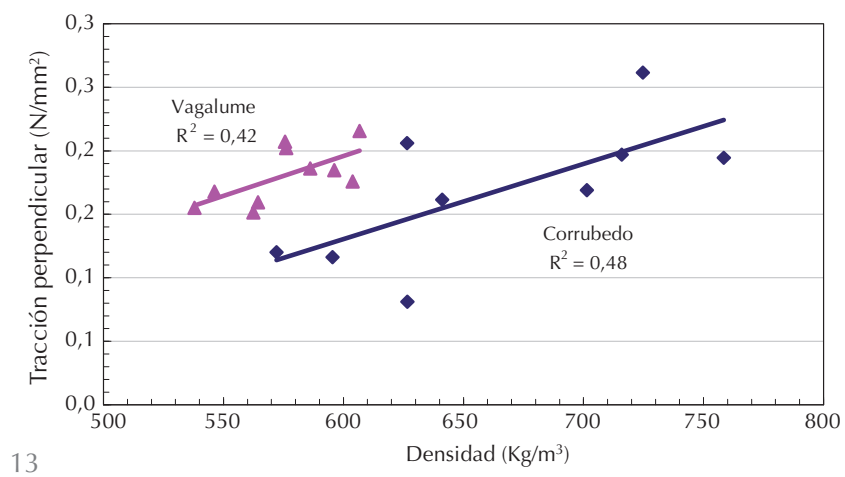

Tabla 8.

Valores de tracción perpendicular a las caras para los tableros de Vagalume y de Corrubedo

\begin{tabular}{|l|r|r|r|r|}
\cline { 2 - 5 } \multicolumn{1}{c|}{} & \multicolumn{2}{c|}{ Vagalume } & \multicolumn{2}{c|}{ Corrubedo } \\
\cline { 2 - 5 } \multicolumn{1}{c|}{} & $\begin{array}{c}\text { Tracción } \\
\left(\mathrm{N} / \mathrm{mm}^{2}\right)\end{array}$ & Densidad $\left(\mathrm{kg} / \mathrm{m}^{3}\right)$ & $\begin{array}{c}\text { Tracción } \\
\left(\mathrm{N} / \mathrm{mm}^{2}\right)\end{array}$ & Densidad $\left(\mathrm{kg} / \mathrm{m}^{3}\right)$ \\
\hline Media & 0,18 & 575,57 & 0,17 & 662,39 \\
\hline$\sigma$ & 0,02 & 23,25 & 0,06 & 60,67 \\
\hline $\mathrm{CV}$ & 0,12 & 0,04 & 0,33 & 0,09 \\
\hline
\end{tabular}

Trabajos previos han obtenido resultados constantes de humedad de equilibrio en tableros de vermiculita expandida aglomerados con silicatos, en un intervalo de variación de humedad relativa del aire del 10\% al 80\% (7). Tanto la hinchazón tras inmersión en agua durante 24 horas (Figura 11 y Tabla 6), como las dos propiedades de resistencia a flexión y a tracción (Figuras 12, 13 y Tabla 7, 8), se incrementan al aumentar la densidad de los tableros estudiados. Consi-

\section{Perfiles de densidad}

Al examinar cuatro probetas individuales de cada material con el perfilómetro, se obtienen unos perfiles que no muestran la forma típica de "valle y pico" característica de algunos tableros derivados de la madera, como es el caso de los tableros convencionales de partículas (25). La probeta analizada del tablero de corteza muestra una densidad media de 594,60 kg/m³ , con un 

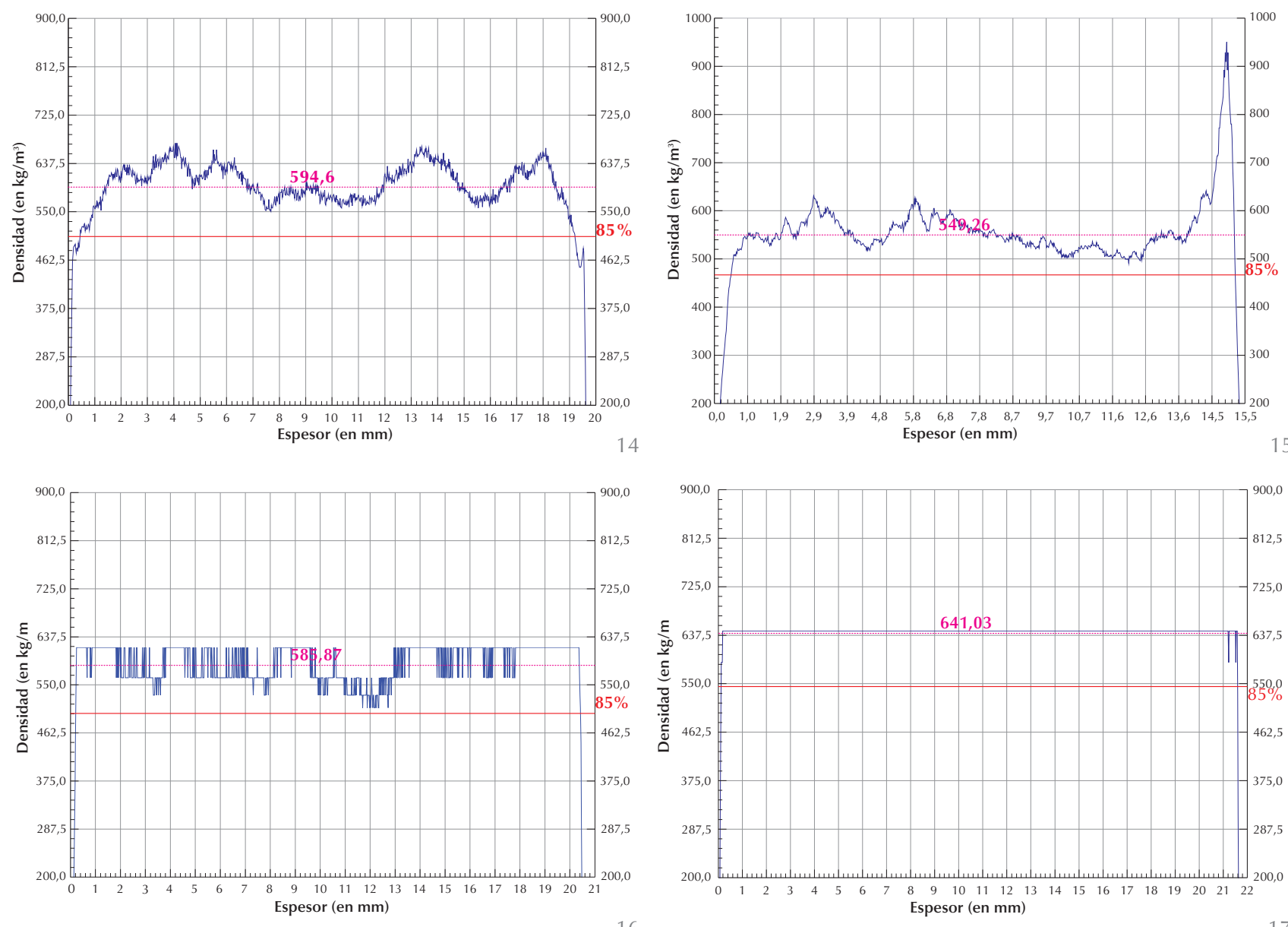

valor máximo de $675,01 \mathrm{~kg} / \mathrm{m}^{3}$ (Figura 14). En la probeta analizada del tablero de virutas, el valor medio obtenido en el perfil es de $549,26 \mathrm{~kg} / \mathrm{m}^{3}$, con un pico máximo de $950,30 \mathrm{~kg} / \mathrm{m}^{3}$ como consecuencia del recubrimiento superficial que posee en la cara vista (Figura 15). En los tableros minerales, la probeta analizada de Vagalume posee una densidad media en el perfil de $585,87 \mathrm{~kg} / \mathrm{m}^{3}$, con un máximo de 617,90 $\mathrm{kg} / \mathrm{m}^{3}$ (Figura 16). En la probeta analizada del tablero de Corrubedo se obtuvo un valor medio de $641,03 \mathrm{~kg} / \mathrm{m}^{3}$, no siendo posible distinguir valores máximos como consecuencia del menor tamaño de partículas de la vermiculita expandida empleada en su fabricación (Figura 17). Estos valores se aproximan al valor medio de densidad calculado a partir de los valores individuales de peso y volumen de cada una de las probetas ensayadas, un total de 40 por cada tipo de tablero (Tabla 9 y 10). Sin embargo, para el tablero de virutas, el valor medio obtenido para todas las probetas ha sido de $484,41 \mathrm{~kg} / \mathrm{m}^{3}(\mathrm{CV}=0,06)$, inferior al valor obtenido con el perfilómetro que incluye en su cálculo el valor máximo obtenido por el recubrimiento superficial (Figura 15). La densidad de los tableros de Corrubedo es mayor que en los tableros de Vagalume, coincidiendo con trabajos previos en los cuales la densidad de la vermiculita expandida aumenta al decrecer el tamaño de las partículas (17).

Los valores medios obtenidos para todas las densidades medidas se resumen en las tablas 9 y 10.

Tabla 9.

Media de todos los valores de densidad del tablero de corteza y del tablero de virutas de madera

\begin{tabular}{|l|r|r|}
\cline { 2 - 3 } \multicolumn{1}{c|}{} & \multicolumn{1}{c|}{ Corteza } & \multicolumn{1}{c|}{ Virutas } \\
\hline Densidad $\left(\mathrm{kg} / \mathrm{m}^{3}\right)$ & 608,58 & 484,41 \\
\hline$\sigma$ & 62,34 & 29,91 \\
\hline CV & 0,10 & 0,06 \\
\hline
\end{tabular}

Tabla 10.

Media de todos los valores de densidad del tablero de corrubedo y vagalume

\begin{tabular}{|l|r|r|}
\cline { 2 - 3 } \multicolumn{1}{c|}{} & Corrubedo & \multicolumn{1}{c|}{ Vagalume } \\
\hline Densidad $\left(\mathrm{kg} / \mathrm{m}^{3}\right)$ & 643,10 & 575,70 \\
\hline$\sigma$ & 52,82 & 20,41 \\
\hline CV & 0,08 & 0,04 \\
\hline
\end{tabular}

14. Perfil de densidad de los tableros de partículas de corteza.

15. Perfil de densidad de los tableros de virutas de madera.

16. Perfil de densidad de los tableros de vermiculita expandida de Vagalume.

17. Perfil de densidad de los tableros de vermiculita expandida de Corrubedo. 


\section{CONCLUSIONES}

Dentro de cada uno de los cuatro tipos de tableros analizados, se incrementan las propiedades mecánicas al aumentar el valor de densidad. Esta tendencia no es tan clara en cuanto a las propiedades físicas estudiadas, donde intervienen otras variables como la geometría y el tamaño de las partículas empleadas. En todo caso, los tableros con mayor densidad alcanzan un mayor valor medio de HEH pero un menor valor medio de hinchazón tras 24 horas de inmersión.

El tablero comercial de virutas de madera empleado como referencia, está elaborado con partículas muy esbeltas y orientadas según un patrón longitudinal. Esto incrementa su resistencia media a la flexión un $317 \%$ respecto a la obtenida en los tableros de corteza de pino, reduciéndose además su variabilidad. Sin embargo, al ser sometidos a esfuerzos de tracción perpendicular a sus caras, la mayor compactación interna de los tableros de corteza, evidenciada por su perfil de densidad y unos valores medios superiores, incrementa su resistencia media un $533 \%$ respecto al obtenido en los tableros de virutas.

El grado de variabilidad, determinado como coeficiente de variación de los datos obtenidos en los ensayos, es reducido en propiedades físicas como la HEH y la densidad, mientras que aumenta considerablemente en la propiedad de hinchazón en espesor, especialmente en el tablero de corteza $(\mathrm{CV}=0,39)$ en el cual la geometría de las partículas puede influir en los valores obtenidos. En cuanto a las propiedades mecánicas, se observa como el grado de variabilidad obtenido en la resistencia a la flexión es similar para todos los grupos de materiales ensayados, $C V=0,25$ y $\mathrm{CV}=0,29$ para los tableros orgánicos, $\mathrm{CV}=$ 0,13 y $\mathrm{CV}=0,16$ para los tableros minerales, mientras que la dispersión obtenida en los valores de tracción perpendicular es superior en aquellos tableros con mayor densidad media y geometría más granular, de $\mathrm{CV}=0,36$ para el tablero de corteza frente a $\mathrm{CV}=0,28$ para el tablero de virutas, y de $\mathrm{CV}=0,33$ para el tablero Corrubedo frente a $\mathrm{CV}=0,12 \mathrm{del}$ tablero Vagalume. En todos los casos, el grado de variabilidad de los datos obtenidos se incrementa al aumentar la densidad media.

Los tableros minerales de partículas de vermiculita expandida presentan una gran homogeneidad en todo su espesor especialmente el tablero Corrubedo, cuyo perfil de densidad no es capaz de detectar variaciones. Un aumento del $8 \%$ de la densidad media en el tablero Corrubedo frente al Vagalume, debido principalmente a su mayor compactación y a un incremento del $10 \%$ en el aglutinante, permite mejorar su resistencia a flexión en un $73 \%$, a pesar de la geometría más granular de sus partículas. Sin embargo, el valor medio de tracción perpendicular a las caras en ambos tableros es prácticamente el mismo, $0,17 \mathrm{~N} / \mathrm{mm}^{2}$ y $0,18 \mathrm{~N} / \mathrm{mm}^{2}$, respectivamente.

La mayor densidad media de los tableros Corrubedo $\left(643 \mathrm{~kg} / \mathrm{m}^{3}\right)$ y de Corteza $\left(609 \mathrm{~kg} / \mathrm{m}^{3}\right)$, frente a los tableros Vagalume $\left(576 \mathrm{~kg} / \mathrm{m}^{3}\right)$ y de Virutas $\left(484 \mathrm{~kg} / \mathrm{m}^{3}\right)$, hace incrementar sus valores medios de $\mathrm{HEH}$ al mismo tiempo que disminuyen los de hinchazón. De esta forma, la HEH media para el tablero de Corteza es un 55\% superior a la del tablero de Virutas, mientras que su valor medio de hinchazón tras inmersión ha sido un $32 \%$ inferior. La misma tendencia se ha encontrado para los tableros minerales, donde el tablero Corrubedo alcanza un valor de $\mathrm{HEH}$ un $18 \%$ superior al del tablero Vagalume, mientras que su valor medio de hinchazón tras inmersión desciende un $46 \%$.

A la vista de los resultados obtenidos, los nuevos tableros analizados constituyen una alternativa eficiente para su empleo en aplicaciones como falsos techos reticulados, revestimientos de paredes, trasdosados, etc., ya que además de aportar las prestaciones térmicas y acústicas reseñadas en la literatura $(2,6,7$ y 8$)$, presentan un buen comportamiento en ambientes húmedos, así como una hinchazón y unas propiedades mecánicas asumibles para su manejo y colocación en obra.

Los resultados obtenidos muestran la posibilidad de intervenir en el proceso de elaboración de estos tableros, modificando la geometría y el tamaño de las partículas empleadas, su posible elaboración en diferentes estratos, la aplicación de acabados decorativos, etc., lo que permitirá desarrollar productos optimizados para aplicaciones específicas.

Este trabajo de investigación ha sido parcialmente financiado por la Xunta de Galicia, en el marco del proyecto PGIDITO6TMT029E.

\section{BIBLIOGRAFÍA}

(1) Olivares Santiago, M.; Galán Marín, C.; Roa Femández, J.; "Los composites: características y aplicaciones en la edificación", Informes de la Construcción, Vol. 54, n. ${ }^{\circ} 484$, marzo-abril (2003), doi:10.3989/ic.2003.v54.i483. 
(2) Pereiro López, G.: Obtención de nuevos materiales para construcción, Ph. D. Tesis, Universidad de Santiago de Compostela (2004), ISBN: 84-9750-426-7.

(3) Pereiro López, G., Guitián R. F.: Procedimiento de obtención de materiales porosos aglomerados con cementos de periclasa, Pat. PCT No: WO 2006/035091A1 (2006).

(4) AENOR: UNE EN 633:1995 Tableros de partículas aglomeradas con cementos. Deficición y clasificación, Madrid (1995), ICS 79.060.20.

(5) Doppelreiter, M., Neuherz, W., Moser, G.: Wood wool construction element and method for its manufacture, Pat. USA, n. ${ }^{\circ}$ : US2001/00143861 (2001).

(6) Lorenzana, T. y Machimbarrena, M.: "Acoustical research about ecological materials", Procceding of Euronoise 06, Finland (2006).

(7) Akao, M.; Fukuda, Y.; Yamazaki, A.; Inoue, M.; Setsu, K.; Takagi, T.; Rui, K.; Okauchi, M. y Tamamura, R.: "Preparation and characterization of Calcium Silicate/Vermiculite composite", Journal Hard Tissue Biology, 15 [3] (2006), pp. 101-104, doi:10.2485/jhtb.15.101.

(8) Pereiro López, G.: "Fomento de la eficiencia a través del empleo de materiales de nueva generación. Un Standard para la Eficiencia Energética". Programa de Promoción Científica. KNX International Forum 10, Madrid (2010), pp. 6-15.

(9) EUWID: EPF planning to draw up its own formaldehyde emissions standard, Europäischer Wirtschaftsdienst WPP, text n. ${ }^{\circ} 001$ (2008).

(10) Moslemi, A. A.: "Emerging technologies in mineral-bonded wood and fiber composites", Advanced Perfor. Materials, vol. 6 (1999), pp. 161-179, doi: 10.1023/A:1008777812842.

(11) Yu, I. A.; Restuccia, G.; Tokarev M. M.; Buerger H. D. y Freni A.: Selective Water Sorbents for Multiple Applications. 11. CaCl2 Confined to Expanded Vermiculite. React. Kinet. Catal. Lett., vol. 71, n. ${ }^{\circ} 2$ (2000), pp. 377-384, doi 10.1023/A:1010351815698.

(12) Mladenovic, A.; Suput, J. S., Ducman, V. y Skapin, A. S.: "Alkali-silica reactivity of some frequently used lightweight aggregates". Cement and Concrete Research, vol. 34 (2004), pp. 1809-1816, doi: 10.1016/j.cemconres.2004.01.017.

(13) Juliá Sanchis, E.: Modelización, simulación y caracterización acústica de materiales para su uso en acústica arquitectónica, Ph. D. Tesis, Universidad Politécnica de Valencia (2008), identificador universal: http://hdl.handle.net/10251/2932 .

(14) Asensio Cerver, F. y Mas, A. M.: ATRIUM I: Materiales para la construcción y sus aplicaciones, vol. 1, pp. 121-129 (1989), ISBN: 84-7741-085-2.

(15) Margarida, M.: "Aislamiento Térmico: Aplicaciones en la Edificación y la Industria. Economía de Energía", Editores Técnicos Asociados (1984), pp. 259-260, ISBN 8471462435

(16) Akao, M.; Yamazaki, A. y Fukuda, Y.: "Vermiculite board for novel building material". Journal of Materials Science, vol. 22 [21] (2003), pp. 1483-1485(3), doi: 10.1023/ A:1026138828315.

(17) Suvorov, S. A. y Skurikhin, V. V.: "Vermiculite- A promising material for high-temperature heat insulators", Refractories and Industrial Ceramics, vol. 44 [3] (2003), pp. 44-52, doi: 10.1023/ A:1026312619843.

(18) AENOR: UNE EN 323:1994. Tableros derivados de la madera. Determinación de la densidad, Madrid (1994), ICS 79.060.01.

(19) AENOR: UNE EN 317:1994 Tableros de partículas y tableros de fibras. Determinación de la hinchazón en espesor después de inmersión en agua, Madrid (1994), ICS 79.060.20.

(20) AENOR: UNE EN 310:1994 Tableros derivados de la madera. Determinación del módulo de elasticidad en flexión y de la resistencia en flexión, Madrid (1994), ICS 70.060.01.

(21) AENOR: UNE EN 319:1994 Tableros de partículas y tableros de fibras. Determinación de la resistencia a la tracción perpendicular a las caras del tablero, Madrid (1994), ICS 79.060.20.

(22) Zapata, N. C.: "Efecto de la mezcla de Pinus radiata y especies nativas sobre la expansión lineal en tableros OSB". M.Sc., Universidad de Valdivia (2004).

(23) T.V.A.: "The Vermiculite Association", Web site: http://www.vermiculite.org/properties. htm, (2009).

(24) Sjöström, E.: Wood chemistry. Fundamentals and Applications, 2. ${ }^{a}$ Ed., Academic Press, (1993), pp. 109-113, ISBN 0126474818.

(25) Poblete, H.; Loncomilla, E. y Inzunza, L.: "Densidad del tablero de partículas y estabilidad dimensional", El Bosque, vol. 21[2] (2000), pp. 69-83, ISSN 0717-9200.

(26) Moslemi, A.: Particleboard Vol. 1: Materials, vol. 1 (1974), p. 239, ISBN: 0809306557.

(27) Shuler, C. y Kelly, R.: "Effect of flake geometry on mechanical properties of eastern spruce flake type particleboard", Forest Products Journal, vol. 26 [6] (1976), pp. 24-28, ISSN 00157473.

(28) Zhou, Y. y Kamdem, P. D.: "Effect of cement/wood ratio on the properties of cementbonded particleboard using CCA-trated wood removed from service", Forest Products Journal, vol. 52 [3] (2002), pp. 77-81, ISSN 00157473.

(29) Brumbauch, J.: "Effect of flake dimension on properties of particleboards". Forest Product Journal, vol. 10[5] (1960), pp. 243-246, ISSN 00157473.

(30) Frybort, S.; Mauritz, R.; Teischinger, A. y Müllera, U.: "Cement bonded composites review", BioResources, vol. 3 [2] (2008), pp. 602-626, ISSN: 1930-2126. 